\title{
Performance Study of FLL Schemes for a Successful Acquisition-to-Tracking Transition
}

\author{
Myriam FOUCRAS, Bertrand EKAMBI \\ ABBIA GNSS Technologies \\ Toulouse, France \\ myriam.foucras@abbia.fr
}

\author{
Ulrich NGAYAP, Jen Yu LI, Olivier JULIEN, \\ Christophe MACABIAU \\ Ecole Nationale de l'Aviation Civile (ENAC) \\ Toulouse, France
}

\begin{abstract}
This article focuses on the analysis of several FLL schemes for different GNSS signals in order to success the acquisition-to-tracking transition at low received $C / \mathbf{N}_{0}$. In a first part, a discussion on different FLL discriminators is lead by comparing frequency error response, linear region, data bit transition insensitivity... Then, based on Monte-Carlo simulations, for each signal, the choices of FLL scheme parameters as the discriminator, loop bandwidth, correlator outputs combination technique are studied in order to highlight the FFL schemes which provide the best performance in terms of probability to get locked.
\end{abstract}

\section{Keywords-FLL; Frequency Tracking Performance}

\section{INTRODUCTION}

In GNSS receivers, the acquisition is the first stage of the signal processing. It consists in assessing the presence of the GNSS signal and providing a rough estimation of the incoming Doppler frequency and code delay. For serial search acquisition method (the classic one), the uncertainty search space is discretized into an acquisition grid. One can easily understand that the choice of the acquisition grid should be the result of a compromise between the acquisition sensitivity and the acquisition execution time. Indeed, the greater the number of bins in the acquisition grid is, the lower the error due to the discretization is but the higher acquisition grid exploration time is. As a consequence, the frequency estimate provided by the acquisition process is generally accurate to within a few tens or hundreds of $\mathrm{Hz}$.

Once acquisition is successful, the tracking stage aims at finely synchronizing the local replica with the incoming signal in order to be able to demodulate it and compute the pseudorange measurements. To do so, a Delay Lock Loop (DLL) and a Phase Lock Loop (PLL) are used to track the code delay and phase respectively. However, at the initiation of the tracking, a refinement on the Doppler frequency is required in order to ensure locking the PLL. One solution is to use a Frequency Lock Loop (FLL) which refines the estimation of the Doppler frequency. It is a critical stage since if this transition is not well calibrated, even a successful acquisition can lead to unsuccessful tracking, especially at low received $\mathrm{C} / \mathrm{N}_{0}$.

The aim of this paper is thus to investigate and present some results on performance of FLL schemes in order to ensure successful acquisition-to-tracking transition. The settings of the FLL have to take into account that data and secondary code demodulation has not yet been achieved.

This analysis will target two GNSS signals: GPS L1 C/A and Galileo E1 OS. For each signal, different FLL configurations are studied. The difference is based on the choice of the FLL parameters such as the discriminator, loop bandwidth, correlator outputs combination techniques... The objective is to compare several configurations per signal to provide the best configuration for each one. The criterion of comparison is the percentage of successful lock. This study takes place in the context of the development of a single frequency GNSS software receiver which aim is to acquire (and then track) L1 signals at $27 \mathrm{~dB}-\mathrm{Hz}$.

The work is mainly divided in two steps. The first consists in studying the theoretical properties of each FLL discriminator. The second step consists in performing MonteCarlo simulations to give the FLL convergence rate.

This article is divided into the following sections.

- A first section aims at establishing the basis by the presentation of the considered GNSS signals and the acquisition process.

- In a second section, the main four FLL discriminators are reviewed.

- Then, the different FLL schemes for simulation are discussed

- In the following section, the Monte-Carlo simulation results are presenting leading to compare FLL schemes for each signal and the probability to success the acquisition-to-tracking transition.

- Finally, conclusions are drawn in the last section.

\section{GNSS SIGNAL AND ACQUISITION MODELS}

In this study, two GNSS signals are considered: the civil GPS and Galileo signals in the L1 band.

\section{A. Signal Model}

The received GPS L1 C/A signal can be modelled at the intermediate frequency as [1]:

$s(t-\tau)=A d(t-\tau) c(t-\tau) \cos \left(2 \pi\left(f_{I F}+f_{D}\right) t+\phi_{0}\right)$

$$
+n(t)
$$


Where

- $A=\sqrt{2 P}$ is the amplitude of the incoming signal at the correlator input and $P$ is the total received power of the signal

- $\quad c$ is the spreading code. There are as many spreading codes as satellites. They have a chipping rate equal to $1.023 \mathrm{MHz}$ and a length of 1023 chips. The spreading code period is $T_{c}=1 \mathrm{~ms}$

- $\quad d$ represents the navigation message with a rate of 50 $\mathrm{b} / \mathrm{s}$. Then, the data bit duration is $T_{d}=20 \mathrm{~ms}$

- $f_{I F}$ is the intermediate frequency of the receiver

- $f_{D}$ is the incoming Doppler frequency

- $\tau$ is the delay of the incoming signal

- $\phi_{0}$ is the initial phase of the incoming signal

- $n$ is the incoming noise which is assumed to be a white noise with centered Gaussian distribution with a constant two-sided power spectral density equal to $N_{0} / 2 \mathrm{dBW}-\mathrm{Hz}$

The GPS L1 C/A signal is a single component signal. As the new GNSS civil signals, the Galileo E1 OS signal has 2 components. The expression of the received Galileo E1 OS signal is [2]:

$$
\begin{aligned}
s(t-\tau)=A\left(\begin{array}{c}
d(t-\tau) c_{1, D}(t-\tau) p_{C B O C, D}(t-\tau) \\
-c_{2}(t-\tau) c_{1, P}(t-\tau) p_{C B O C, P}(t-\tau)
\end{array}\right) \\
\times \cos \left(2 \pi\left(f_{I F}+f_{D}\right) t+\phi_{0}\right)+n(t)
\end{aligned}
$$

Where

- $A=\sqrt{P}$ is the amplitude of the incoming signal at the correlator input on each component

- $\quad c_{1, D}$ and $c_{1, P}$ are the spreading codes on the data and pilot component. Their chipping rates are the same as GPS L1 C/A ones but their length are 4092 chips.

- $\quad c_{2}$ on the pilot component is a known secondary code of 25 bits and transmitted at $250 \mathrm{~b} / \mathrm{s}$ (as the navigation message on the data component). The spreading code period $T_{c}$ is the same as the duration of the data and secondary code bits (4 ms)

- $\quad p_{C B O C, D}$ and $p_{C B O C, P}$ are the sub-carriers carried by the data and pilot components

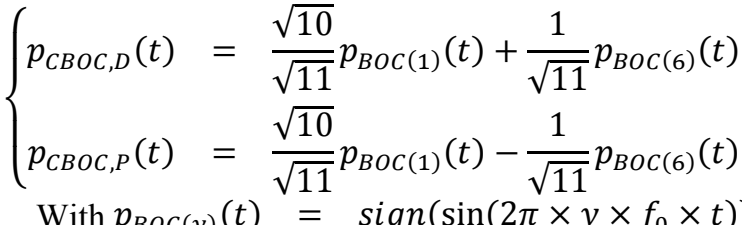

With $p_{B O C(y)}(t)=\operatorname{sign}\left(\sin \left(2 \pi \times y \times f_{0} \times t\right)\right)$

The GPS L1 C/A spreading code period is 20 times shorter than the data bit duration which constitutes a notable difference regarding the Galileo E1 OS signal -data and secondary code bit sign transitions can occur at each spreading code period with a probability of one half, independently. This difference in signal structure could suggest differences in frequency tracking performance.

\section{B. Correlator Output Model}

GNSS receivers generate a local replica of the incoming signal depending on an estimation of the code delay $\hat{\tau}$ and of the Doppler frequency $\widehat{f_{D}}$. The correlation process between the incoming signal and the local replica provides in-phase $I$ and quadrature $Q$ correlator outputs.

For Galileo E1 OS, four different correlator outputs are considered; two per component, as presented in [3]. For GPS L1 C/A, only two correlator outputs are computed, they correspond to the data correlator outputs (4):

$$
\begin{aligned}
& I_{x}(k)=\frac{A_{x}}{2} R_{x}\left(\varepsilon_{\tau}\right) \cos \left(\pi \varepsilon_{f} T_{I}+\varepsilon_{\phi_{0}}\right) \operatorname{sinc}\left(\pi \varepsilon_{f} T_{I}\right)+n_{I_{x}}(k) \\
& Q_{x}(k)=\frac{A_{x}}{2} R_{x}\left(\varepsilon_{\tau}\right) \sin \left(\pi \varepsilon_{f} T_{I}+\varepsilon_{\phi_{0}}\right) \operatorname{sinc}\left(\pi \varepsilon_{f} T_{I}\right)+n_{Q_{x}}(k) \\
& \quad \text { Where }
\end{aligned}
$$

- $\quad x$ stands for " $D$ " for the data component or " $P$ " for the pilot component

- $d_{x}$ is the data sequence on the data component or the secondary code on the pilot component

- $\varepsilon_{\tau}=\tau-\hat{\tau}$ is the code delay error

- $\varepsilon_{f}=f_{d}-\widehat{f_{d}}$ is the Doppler frequency error

- $\varepsilon_{\phi_{0}}=\phi_{0}-\widehat{\phi}_{0}$ is the initial phase error at the beginning of the correlation interval

- $n_{I}$ and $n_{Q}$ are the noises at the correlator output which follow a centered Gaussian distribution with variances $\sigma^{2}=\frac{N_{0}}{4 T_{I}}[4]$

- $\left[T_{0} ; T_{0}+T_{I}\right]$ is the integration interval beginning at $T_{0}$ and lasting $T_{I}$. The coherent integration time is chosen to be a spreading code period, this means $T_{I}=1 \mathrm{~ms}$ for GPS L1 C/A, $T_{I}=4 \mathrm{~ms}$ for Galileo E1 OS

- $\quad R_{D}$ and $R_{P}$ are the autocorrelation functions on the data and pilot component respectively

Note that the above correlator outputs model neglects the cross-correlation between the data and pilot component because the spreading codes were chosen to be as orthogonal as possible.

\section{Acquisition Process}

The acquisition consists in giving a rough estimation of the incoming parameters. A classical acquisition method, the serial search acquisition method is based on an acquisition grid which covers the frequency and time uncertainty search spaces. As it is done in [5], in the frequency domain, when considering that the maximum degradations due to the Doppler discretization should be lower than $0.9 \mathrm{~dB}$. The Doppler bin size should be chosen equal to $\frac{1}{2 T_{I}} \mathrm{~Hz}$ resulting in a Doppler error in the window $\left[-\frac{1}{4 T_{I}} ; \frac{1}{4 T_{I}}\right] \mathrm{Hz}$.

\section{Frequency Lock Loop}

The Frequency Lock Loops (FLL) are used in a wide range of communication systems as a means of achieving carrier synchronization [6]. In the area of GNSS signal 
processing, they are used to track the Doppler frequency of the incoming signal which is dominated by the satellite-toreceiver motion and the user clock drift [7]. It is well known the FLL is more robust than a PLL that aims at tracking the phase of the incoming signal. The FLL plays thus a key role in facilitating reliable signal tracking.

The FLL tracks the carrier frequency by computing a frequency error estimate between the received and the local signals. As any other classical feedback control loop, this estimate is filtered by a low-pass filter and then used to control a Numerically Control Oscillator (NCO). A simplified block diagram of this operation is depicted in Fig. 1.

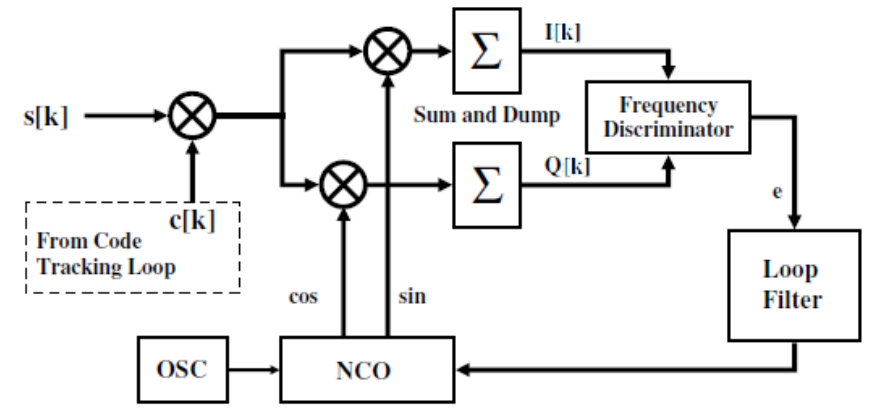

Fig. 1: Block diagram of a typical frequency lock loop [7]

The carrier frequency error can be computed using the difference in power [8], [10] or as it will be studied in this work by means of frequency discriminators. A frequency discriminator is a function which produces an estimate of the frequency error from two consecutive correlator outputs (I/Q) pairs. This approach estimates the phase error at each epoch and then the frequency error is the phase variation per integration interval:

$$
\varepsilon_{\phi}(k)-\varepsilon_{\phi}(k-1)=2 \pi \varepsilon_{f}(k) T_{I}
$$

Where $\varepsilon_{\phi}(k)$ is the phase error at the instant $k$

Generally, frequency discriminators can produce an accurate estimate only over a limited region, called the linear region which is inversely proportional to the integration time.

\section{CONSIDERED FREQUENCY DISCRIMINATORS}

In this section, four popular frequency discriminators are analyzed by summarizing their main characteristics. For more details, the reader is invited to read [7] for an overview of all of the discriminators or [10] and [11] for the Cross Product and Differential Arctangent discriminators and [12] and [13] for the Decision Directed Cross Product and Four Quadrant Arctangent discriminators.

The below analysis on the FLL discriminators is presented for only one component, without loss of generalities, the data component. Clearly, the results are applicable to the pilot component by substituting the data bit $d(k)$ by the secondary code bit $c_{2}(k)$ because the two sequences (data and secondary code for Galileo E1 OS) have the same bit rate. There is no loss on the acquisition detector amplitude due to the well estimation of the code delay
$\left(R\left(\varepsilon_{\tau}(k)\right)=1\right)$. Based on this, the correlator outputs given in (4) become:

$$
\begin{aligned}
I(k) & =\frac{A}{2} d(k) \cos \left(\varepsilon_{\phi}(k)\right) \operatorname{sinc}\left(\pi \varepsilon_{f}(k) T_{I}\right)+n_{I}(k) \\
Q(k) & =\frac{A}{2} d(k) \sin \left(\varepsilon_{\phi}(k)\right) \operatorname{sinc}\left(\pi \varepsilon_{f}(k) T_{I}\right)+n_{Q}(k)
\end{aligned}
$$

With $\varepsilon_{\phi}(k)=\pi \varepsilon_{f}(k) T_{I}+\varepsilon_{\phi_{0}}(k)$

Let us note that in the context of this work, we assume that the incoming frequency $f_{D}$ is constant.

\section{A. Cross Product Discriminator}

The first presented discriminator, is the Cross Product discriminator (noted as $C P$ ) and defined as:

$$
\begin{gathered}
e_{C P}(k)=\frac{1}{T_{I}}(Q(k) I(k-1)-I(k) Q(k-1))= \\
A_{C P} d(k-1) d(k) \frac{\sin \left(\varepsilon_{\phi}(k)-\varepsilon_{\phi}(k-1)\right)}{T_{I}}+n_{C P}(k)
\end{gathered}
$$

Where

- $A_{C P}=\frac{A^{2}}{4} \operatorname{sinc}\left(\pi \varepsilon_{f}(k-1) T_{I}\right) \operatorname{sinc}\left(\pi \varepsilon_{f}(k) T_{I}\right)$ is the amplitude term where the product of the sinc can be approximated by $\operatorname{sinc}^{2}\left(\pi \varepsilon_{f}(k) T_{I}\right)$

- $n_{C P}(k)$ is the output noise, described in Appendix A

The output frequency error is a sine function of the consecutive phase errors and depends on the signal amplitude. Then, the frequency error estimate produced by the cross product discriminator must be normalized by $\frac{A^{2} \pi}{2}$ but this normalization does not pose a significant problem [7]. Secondly, it is evident from (7) that the sign of frequency estimate depends on the data bit signs associated to the consecutive correlator outputs. That means that the frequency estimate would be inverted each time that $d(k)$ differs from $d(k-1)$ which would lead to significant performance degradation. Then, care must be taken when applying it to data modulated signals.

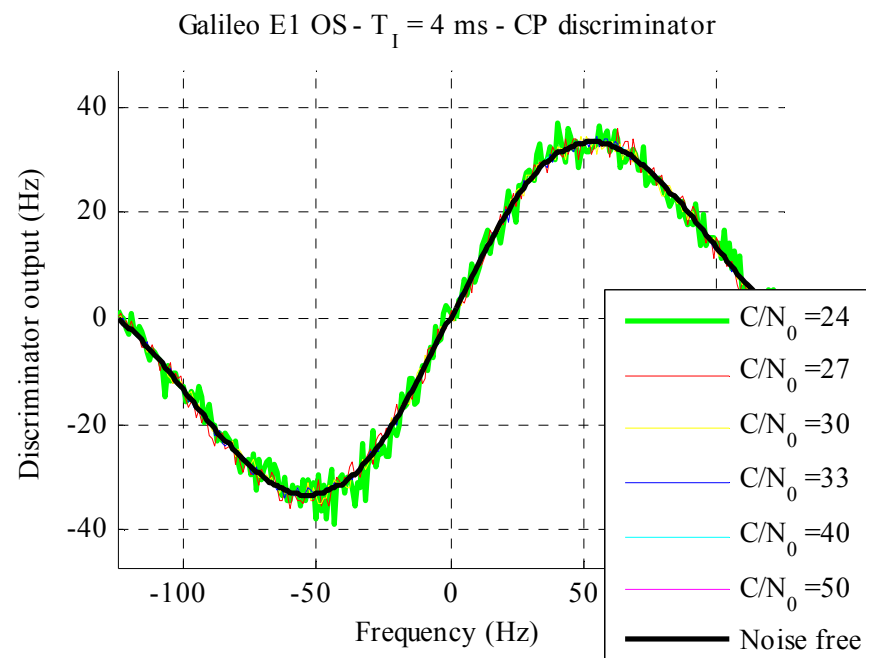

Fig. 2: $\mathrm{CP}$ discriminator response to frequency error for a noise-free signal and different $\mathrm{C} / \mathrm{N}_{0}$ cases 
From Fig. 2 for Galileo E1 OS (and in Appendix A Fig. 21 for GPS L1 C/A) which represents the mean discriminator response to frequency error, it can be observed that its shape does not depend upon the $\mathrm{C} / \mathrm{N}_{0}$. It is also one of the most computationally efficient discriminator.

The linear region (where the black curve representing the noise-free case can be approximated by a line) is approximately by $[-30 ; 30] \mathrm{Hz}$ for Galileo E1 OS.

\section{B. Decision Directed Cross Product Discriminator}

As previously seen, the Cross Product discriminator is affected by data bit transitions. If there is a data bit sign transition, the Cross Product discriminator results in the wrong sign. The Decision Directed Cross Product, noted as DDCP, solves the problem by modulating the Cross Product discriminator with the sign of the dot product:

$$
\begin{gathered}
e_{D D C P}(k) \\
=e_{C P}(k) \times \operatorname{sign}(I(k) I(k-1)+Q(k) Q(k-1))
\end{gathered}
$$

Without noise, (8) can be shown to be equivalent to (9). Then, the estimated frequency error is a sine function of twice the difference of phase errors.

$$
e_{D D C P}(k)=A_{C P} \times \frac{\sin \left(2\left(\varepsilon_{\phi}(k)-\varepsilon_{\phi}(k-1)\right)\right)}{T_{I} \times\left|\cos \left(\varepsilon_{\phi}(k)-\varepsilon_{\phi}(k-1)\right)\right|}
$$

The response for the noise-free case (in Fig. 3 for Galileo E1 OS and Fig. 22 for GPS L1 C/A) is similar to that of the Cross Product discriminator but it also exhibits sign inversions due to the sign term, the linear region being the same. As it is shown, unlike the cross product discriminator, the mean response is sensitive to the $\mathrm{C} / \mathrm{N}_{0}$. Indeed, the term $\operatorname{sign}(D o t)$ gives rise to the sensitivity dependency of the discriminator since it should give the sign of the product of the consecutive data bits contained in the consecutive correlator outputs but due to thermal noise, it provides the opposite sign. Galileo E1 OS - $\mathrm{T}_{\mathrm{I}}=4 \mathrm{~ms}$ - DDCP discriminator

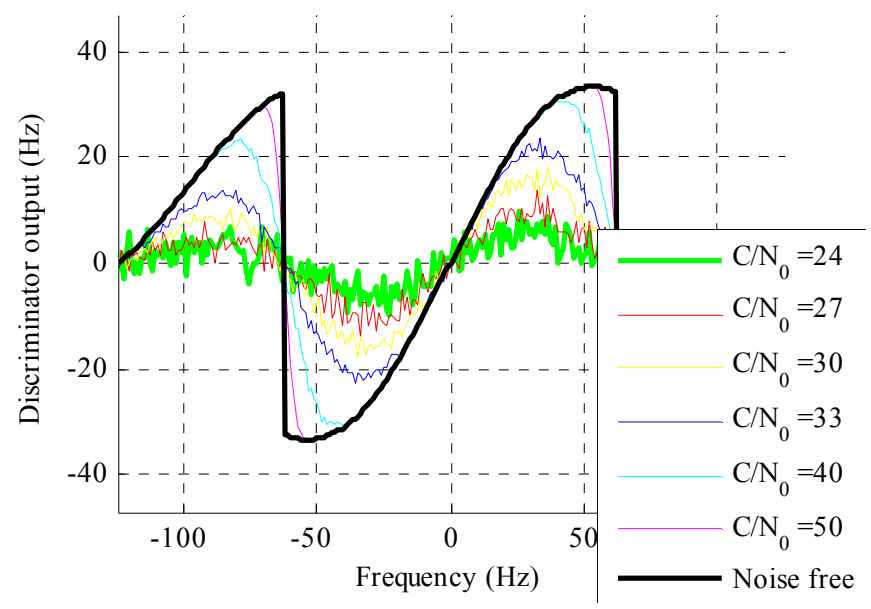

Fig. 3: DDCP discriminator response to frequency error for a noise-free signal and different $\mathrm{C} / \mathrm{N}_{0}$ cases

\section{Differential Arctangent Discriminator}

One of the optimal phase estimators in the Maximum Likelihood sense is $\widehat{\varepsilon_{\phi}}(k)=\tan ^{-1}(Q(k) / I(k))$. Then, based on this, a frequency discriminator can be computed as the difference of the phase estimator at two consecutive instants [11].

$$
e_{\text {Atan }}(k)=\frac{1}{2 \pi T_{I}} U\left(\tan ^{-1}\left(\frac{Q(k)}{I(k)}\right)-\tan ^{-1}\left(\frac{Q(k-1)}{I(k-1)}\right)\right)
$$

where $U$ is a phase-unwrapping function which maps the phase estimate to the interval $\left[-\frac{\pi}{2} ; \frac{\pi}{2}\right]$ as defined in Appendix C. This discriminator presents the advantage as the DDCP discriminator to be insensitive to data bits.

Galileo E1 OS - $\mathrm{T}_{\mathrm{I}}=4 \mathrm{~ms}$ - Atan discriminator

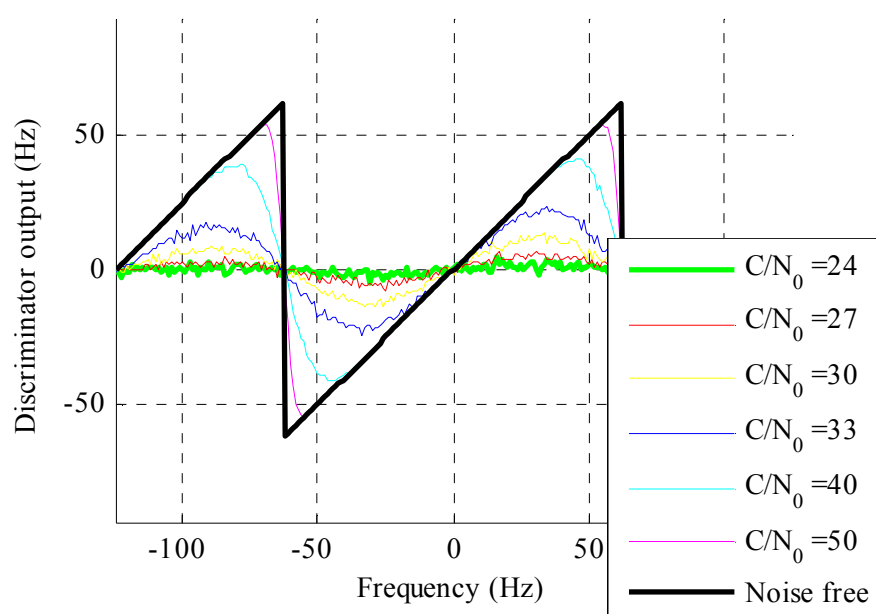

Fig. 4: Atan discriminator response to frequency error for a noise-free signal and different $\mathrm{C} / \mathrm{N}_{0}$ cases

As depicted in Fig. 4 and Fig. 23, the linear region of the Differential Arctangent discriminator is $\left[-\frac{1}{4 T_{I}} ; \frac{1}{4 T_{I}}\right]$.

\section{Four-Quadrant Arctangent (Atan2) Discriminator}

The last presented discriminator is the Atan2 discriminator which is self-normalized as the Differential Arctangent discriminator. This discriminator, as we can see with its expression (11) given without noise is sensitive to data modulation. Indeed, the denominator is always positive for relative small phase error, then the sign of $e_{\text {Atan2 }}(k)$ depends on the sign of $d(k-1) d(k)$.

$$
\begin{aligned}
& e_{\text {Atan } 2}(k)=\frac{1}{2 \pi T_{I}}(\operatorname{atan} 2(C r o s s, D o t)) \\
= & \frac{\tan ^{-1}\left(\frac{d(k-1) d(k) \sin (\phi(k)-\phi(k-1))}{1+d(k-1) d(k) \cos (\phi(k)-\phi(k-1))}\right)}{2 \pi T_{I}}
\end{aligned}
$$

Let us remark that as it is done in [13], the notation used here is $\operatorname{atan} 2$ (Cross, Dot) to have directly the tangent term as decribed in Appendix D but some references [12], [6] give $\operatorname{atan} 2(D o t, C r o s s)$ which is equivalent because for all $x$ in $\mathbb{R}^{*+}, \tan ^{-1}\left(\frac{1}{x}\right)+\tan ^{-1}(x)=\frac{\pi}{2}$.

From Fig. 5 for Galileo E1 OS and Fig. 24 for GPS L1 $\mathrm{C} / \mathrm{A}$, the linear region is the largest compared to the previous 
discriminators and it is evident that the response is also sensitive to the received $\mathrm{C} / \mathrm{N}_{0}$.

Galileo E1 OS - $\mathrm{T}_{\mathrm{I}}=4 \mathrm{~ms}$ - Atan2 discriminator

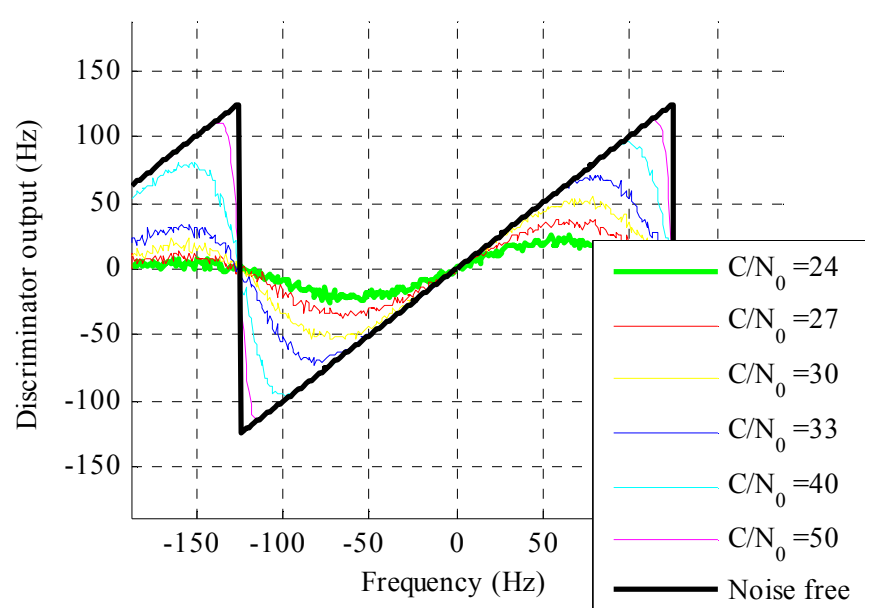

Fig. 5: Atan2 discriminator response to frequency error for a noise-free signal and different $\mathrm{C} / \mathrm{N}_{0}$ cases

\section{E. Discussion}

1) Linear region and discriminator gain

For a frequency discriminator, the linear region is the frequency for which the noise-free curve has a linear shape. As previously said, for most of the discriminators, the linear region changes with changing $\mathrm{C} / \mathrm{N}_{0}$. It is worth noting that the linear region should be as large as possible to ensure a better stability of the loop for large errors. For the Cross-Product and Decision Directed Cross Product discriminators, the linear region is $\left[-\frac{1}{8 T_{I}} ; \frac{1}{8 T_{I}}\right]$ whereas it is $\left[-\frac{1}{2 T_{I}} ; \frac{1}{2 T_{I}}\right]$ for the Atan2 discriminator.

The discriminator gain $K_{D}$ is defined as the slope of the discriminator function when the frequency error is zero. A carrier frequency estimate can under certain conditions discussed in [6], be approximated by the gain plus an independent corrupting noise noted $n^{w}$ :

$$
e(k) \approx \frac{1}{T_{I}} K_{D}\left(\varepsilon_{\phi}(k)-\varepsilon_{\phi}(k-1)\right)+n^{w}(k)
$$

Under strong signal power condition, the slope is approximately unity. As the $\mathrm{C} / \mathrm{N}_{0}$ is reduced, the slope of the discriminator function usually becomes smaller, as seen in Fig. 3 for instance. As the relationship is nonlinear, discriminator gains for different $\mathrm{C} / \mathrm{N}_{0}$ are estimated using Monte-Carlo simulations and approximations models. The theoretical gain discriminator gains are given in (13) from [7]. These values were confirmed through simulations as shown in Fig. 6.

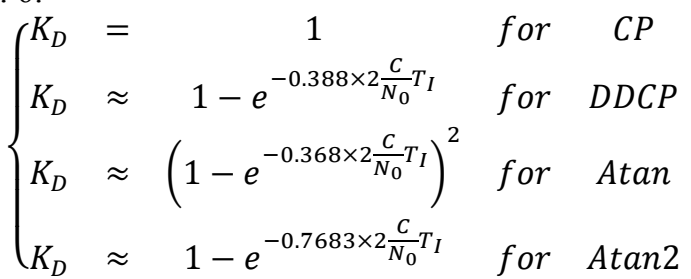

In Fig. 6, the line curves are the representation of the theoretical gains given by (13) and experimental gains, marked by circle, result in the slope computation by hands. It can be observed that they agree well.

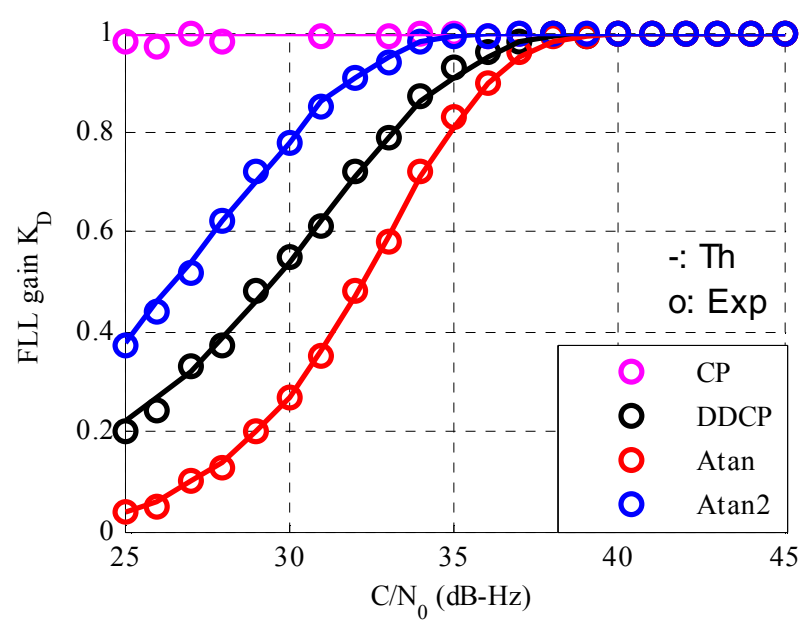

Fig. 6: Theoretical and estimated discriminator gains

\section{2) FLL tracking error}

The dominant sources of frequency error in a FLL are frequency jitter due to thermal noise and dynamic stress error [12]. The FLL tracking loop jitter due to thermal noise is given by [12]:

$$
\sigma_{F L L}=\frac{1}{2 \pi T_{I}} \sqrt{\frac{4 F B_{L}}{C / N_{0}}\left(1+\frac{1}{T_{I} C / N_{0}}\right)}
$$

Where $F$ is a parameter which value is 1 or 2 .

Simulations were run for two discriminators to verify the correct implementation of the FLL. The variance of the FLL frequency error is computed over 10 seconds assuming a null initial error. The FLL bandwidth was chosen equal to $10 \mathrm{~Hz}$. Results are plotted in Fig. 7.

As it can be observed, experimental results (with marker) match perfectly the theoretical results (solid line) for both signals but with two different values for $F$ (1 for GPS L1 C/A and 2 for Galileo E1 OS). The validation of our FLL model permits also to highlight that the tracking error variance is not a linear function of the $\mathrm{C} / \mathrm{N}_{0}[6]$. 


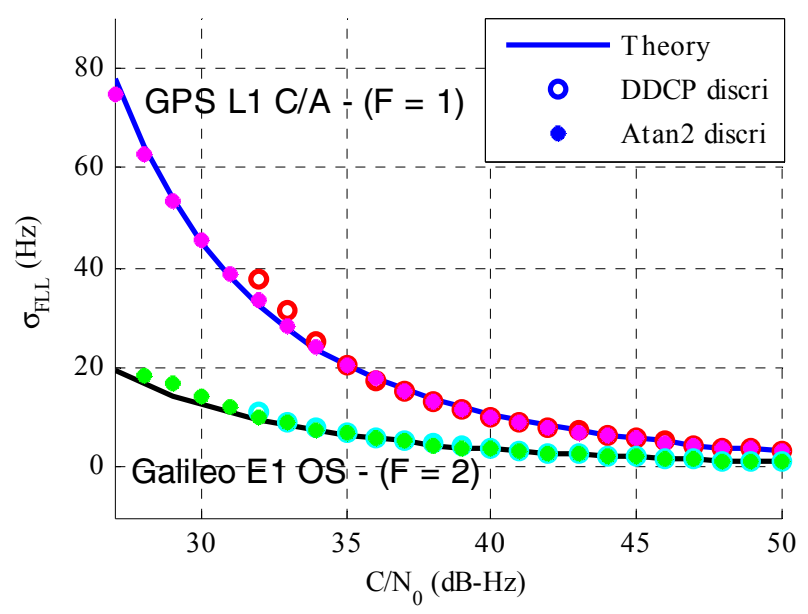

Fig. 7: Frequency error standard deviation due to thermal noise (theoretical and simulated results $)\left(B_{L}=10 \mathrm{~Hz}\right)$

\section{FLL SCHEMES}

\section{A. Simulation inputs}

Monte-Carlo simulations are based on coherent correlator outputs on the spreading code period, that means $1 \mathrm{~ms}$ for GPS L1 C/A and $4 \mathrm{~ms}$ for Galileo E1 OS.

\section{1) Loop bandwidth}

For simulations, the loop order is chosen to be 2 to be able to track jerk dynamics (this is a typical choice for FLLs). The one-sided noise bandwidth $B_{L}$ is defined in [7]. In our study, it can take values between 1 and $10 \mathrm{~Hz}$. A system with a higher bandwidth has a faster response because the convergence time is in the order of $\frac{1}{B_{L}}$ (in seconds) but a smaller steady state error.

\section{2) Correlator combination techniques}

Different correlator combination techniques are proposed and studied to counteract the impact of data and secondary code bit transitions. For simulations, the data and secondary code are implemented. For the GPS L1 C/A signal, the data bit location is randomly chosen among the 20 spreading code periods. In the same way, the first implemented secondary code bit is randomly chosen between the 25 bits.

\section{a) GPSL1 C/A}

- $\quad$ FLL update every $2 \times T_{I}$

This technique is the classical use of a FLL. Two consecutive pairs of correlator outputs are computed and are used for the FLL discriminator computation. The frequency update is based on the FLL discriminator output. The update of the local frequency is thus taking place every two spreading code period. An illustration of this technique is presented in Fig. 8.

Because right after the acquisition, the receiver is not synchronized with the data sequence, the data bit boundaries are not known for the acquisition to tracking transition. This implies that it is possible that, one pair of correlator outputs can straddle two data bits as it is illustrated for correlator outputs 7 and 8 in Fig. 8. This bad configuration occurs only for 1 every 10 correlator output pairs. But the data bit change does not necessary imply a sign change. Data bit transition

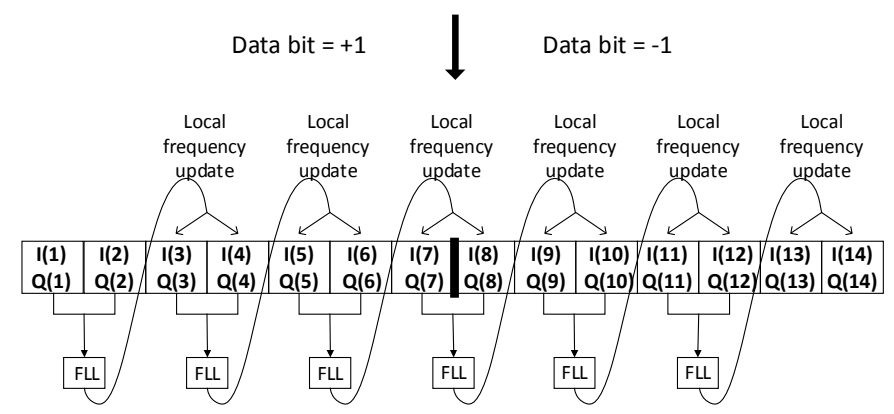

Fig. 8: Classical FLL technique

\section{- $\quad$ FLL update every $20 \mathrm{~ms}$}

The second GPS L1 C/A technique is briefly presented in [11]. Indeed, the discriminator output is in fact an average value on the 19 discriminator outputs computed over a data bit duration is taken which are the first $T_{I} \mathrm{~ms}$ with second $T_{I} \mathrm{~ms}$, second $T_{I}$ ms with third $T_{I}$ ms... The scheme is presented in Fig. 9.

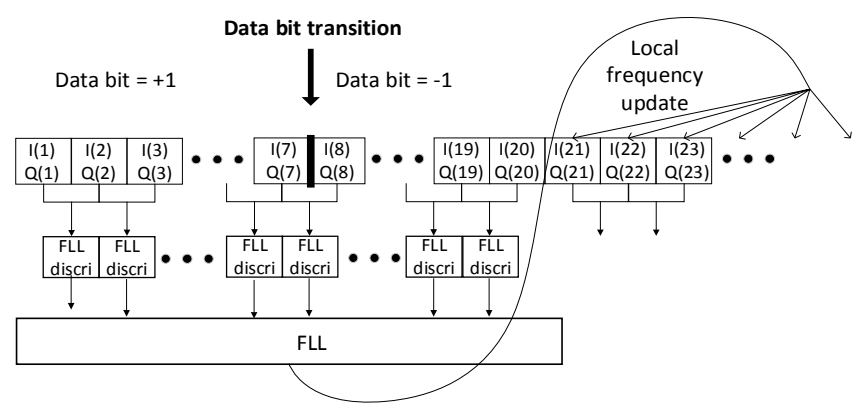

Fig. 9: Second GPS L1 C/A FLL technique

\section{b) Galileo E1 OS}

- FLL update every $2 \times T_{I}$ : One component

The first technique for Galileo E1 OS consists in considering only one component (data or pilot because the secondary code is not acquired). This technique is similar to the first one for GPS L1 C/A.

- FLL update every $2 \times T_{I}$ : Two components

The second technique for Galileo E1 OS consists in considering both components to acquire the total received signal power. The data and pilot components are processed separately and each one provides a FLL discriminator output. The frequency update is based on the average value of the discriminator outputs obtained from the correlation on the data component and on the pilot component.

- Frequency update at $20 \mathrm{~ms}$

To mimic what is done for the GPS L1 C/A in second technique, the update of the local frequency every $20 \mathrm{~ms}$ based on an average discriminator output. Then on both components, the discriminator output is computed for each consecutive pair (4 discriminators outputs per component). 


\section{3) Frequency input}

The uncertainty on the frequency at the initiation of the tracking is equal to half of the width of the Doppler acquisition bin which is $\frac{1}{4 T_{I}}$, as seen in section $C$. It is reasonable to assume that the distribution of the Doppler error at the initiation of the tracking phase is uniform.

To test the dependency of the FLL tracking performance as a function of the initial Doppler error, the initial uncertainty region $\left[-\frac{1}{4 T_{I}} ; \frac{1}{4 T_{I}}\right]$ is split into $N_{f}$ sub-bins. For each sub-bin, 200 uniformly distributed initial frequencies are tested. The simulation output result is thus obtained for each sub-bin and a more general simulation result can be obtained by averaging the results from each sub-bin.

\section{B. Simulation output}

During the simulations, it was decided that the FLL was locked when the frequency error output is in the corresponding discriminator linear region after 20 seconds, which is representative of a convergence (or at least, no divergence). The probability that the FLL gets locked can be seen as the convergence success rate.
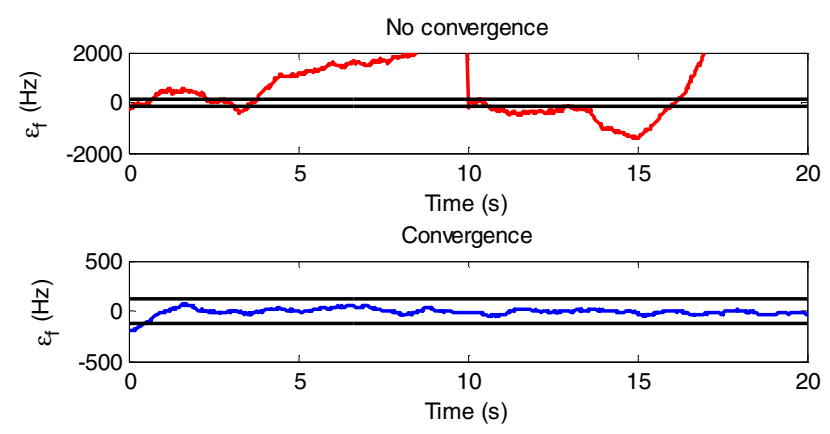

Fig. 10 Frequency error standard deviation due to thermal noise (theoretical and simulated results) for GPS L1 C/A for different bandwidths

Fig. 10 illustrates the convergence notion. For the upper case, there is no convergence because after 20 seconds, the frequency error is not in the linear region materialized by the black lines. In the second case, the frequency error is in the linear region then we consider that there is convergence. The probability to get locked, noted $P_{l}$, is then the number of times that the frequency error is after 20 seconds in the linear region over the number of simulations.

\section{Simulation scheme}

The objective of the simulation part is to determine the best FLL schemes for GPS L1 C/A and Galileo E1 OS to transition from acquisition to PLL tracking. To do so, MonteCarlo simulations are run for different FLL schemes (around 30 for each signal according to 3 varying parameters). 200 simulations were run for each configuration and for 51 frequencies.

\section{Simulations Results}

Monte-Carlo simulations results will be presented in this section. For each signal, the first ones focus on a received total $\mathrm{C} / \mathrm{N}_{0}$ of $27 \mathrm{~dB}-\mathrm{Hz}$ (acquisition threshold). Figures are divided in 4 windows, the first column is for data modulation sensitive discriminators and the first line for Cross Product based discriminators. For each discriminator, 4 curves are plotted, one per tested loop bandwidth.

The second presented results are the probabilities to get locked as a function of the total received $\mathrm{C} / \mathrm{N}_{0}$. In this case, on the same figure, the results, for the four discriminators are plotted. They are obtained by averaging over all possible initial Doppler errors within an acquisition bin, and for a bandwidth of $1 \mathrm{~Hz}$.

\section{A. FLL Scheme for GPS L1 C/A}

\section{1) For a given $\mathrm{C} / \mathrm{N}_{0}$ of $27 \mathrm{~dB}-\mathrm{Hz}$}

From Fig. 11 and Fig. 12, for the "FLL update every $2 \mathrm{~ms}$ " and "FLL update every $20 \mathrm{~ms}$ " techniques, it can be observed that they have the same trend behavior but the second technique (on $20 \mathrm{~ms}$ ) presents better probabilities to get locked. At $27 \mathrm{~dB}-\mathrm{Hz}$, the highest probabilities of detection $\left(P_{l}=1\right)$ is for the CP and Atan2 discriminators. They are both data modulation sensitive but for the GPS L1 C/A signal, this feature is not a crucial issue due to the data duration equal to $20 \mathrm{~ms}$ with a probability to bit sign transition of one half. These results can be explained by the noise independence for the CP discriminator and the large linear region for the Atan2 discriminator. The best probability to get locked is obtained for a loop bandwidth of $1 \mathrm{~Hz}$ but even for high loop bandwidth for Atan 2 and CP discriminators, the probability is still 1. So, depending on time to convergence criteria, a loop bandwidth higher than $1 \mathrm{~Hz}$ can be chosen. On the other hand, the DDCP and Atan do not seem recommended for the acquisition to tracking transition with high loop bandwidths.

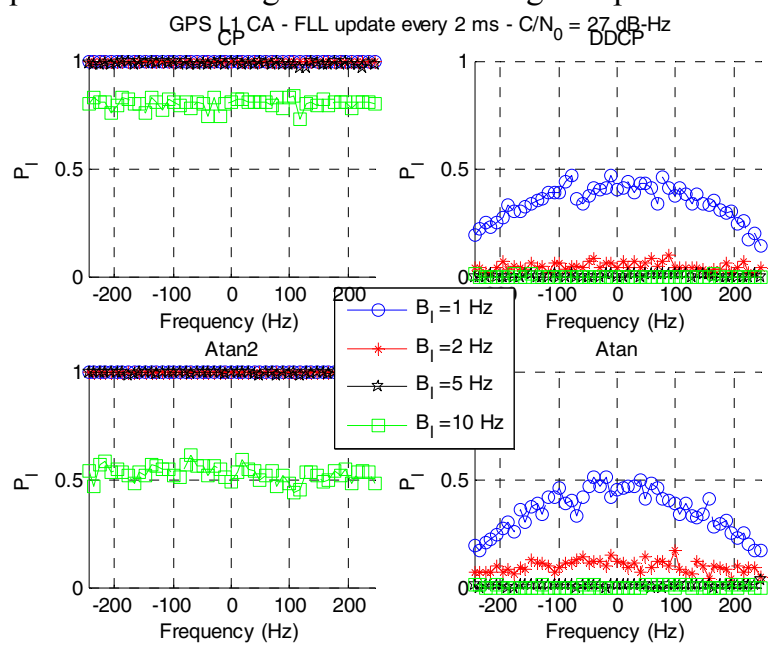

Fig. 11: Results for FLL schemes for GPS L1 C/A at $27 \mathrm{dBHz}$ for the first simulated technique (on $2 \mathrm{~ms}$ ) 


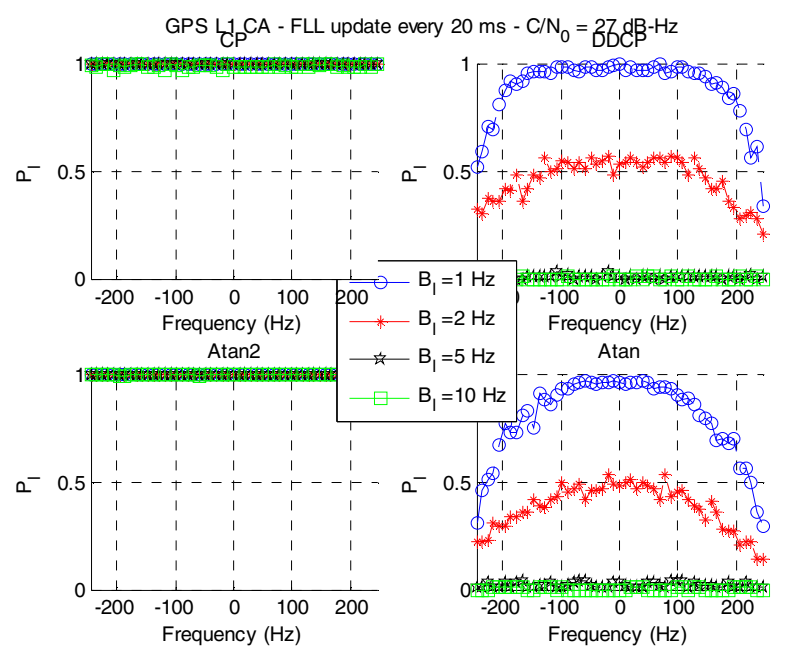

Fig. 12: Results for FLL schemes for GPS L1 C/A at $27 \mathrm{dBHz}$ for the second simulated technique (on $20 \mathrm{~ms}$ )

\section{2) For different $C / N_{0}$}

The DDCP discriminator is not as good as the CP discriminator at $27 \mathrm{~dB}-\mathrm{Hz}$ because unlike the $\mathrm{CP}$ discriminator, it is noise sensitive. This can be checked with Fig. 13 and Fig. 14. The curve representing the DDCP discriminator results (in red dots) increases when the $\mathrm{C} / \mathrm{N}_{0}$ increases. For the GPS L1 C/A signal, only a few correlator outputs can be corrupted by a bad estimation of the data bits product and the associated degradations should not be high but this explains why a probability of successful convergence of 1 is hardly reached.

GPS L1 CA - $B_{L}=1 \mathrm{~Hz}-$ FLL update every $2 \mathrm{~ms}$

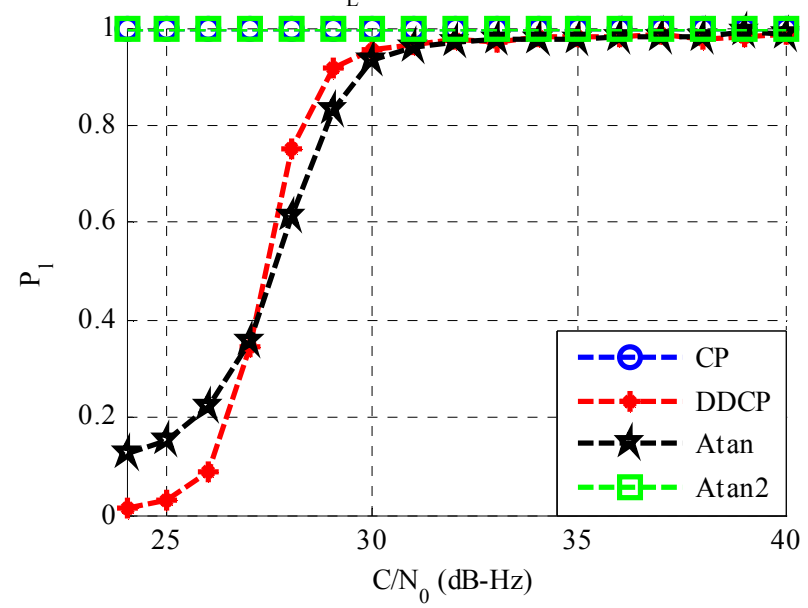

Fig. 13: Results for FLL schemes for GPS L1 C/A for the first simulated technique (on $2 \mathrm{~ms}$ )

To conclude this section on the GPS L1 C/A results, it has been shown that the probability to get locked is 1 even at low $\mathrm{C} / \mathrm{N}_{0}$ for the $\mathrm{CP}$ and Atan2 discriminators. The second technique (FLL update every $20 \mathrm{~ms}$ ) provides an improvement on the average probability of successful convergence for the DDCP discriminator at $27 \mathrm{~dB}-\mathrm{Hz}$ equivalent to a gain of 1.5 $\mathrm{dB}$ (equivalent $\mathrm{C} / \mathrm{N}_{0}$ ). The $\mathrm{CP}$ discriminator presents a probability to get locked at 1 whichever the technique and whichever the sensitivity.

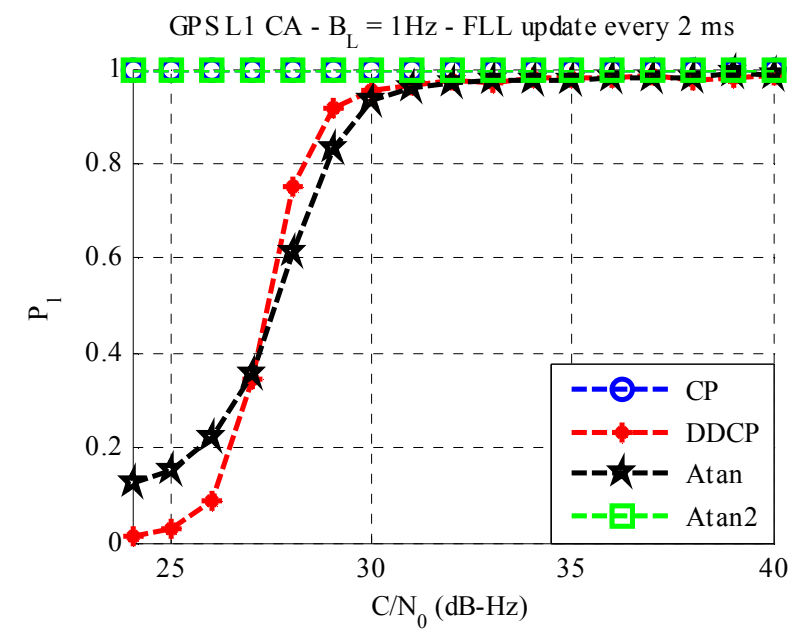

Fig. 14: Results for FLL schemes for GPS L1 C/A for the second simulated technique (on $20 \mathrm{~ms}$ )

\section{B. FLL Scheme for Galileo E1 OS}

1) For a given $\mathrm{C} / \mathrm{N}_{0}$ of $27 \mathrm{~dB}-\mathrm{Hz}$

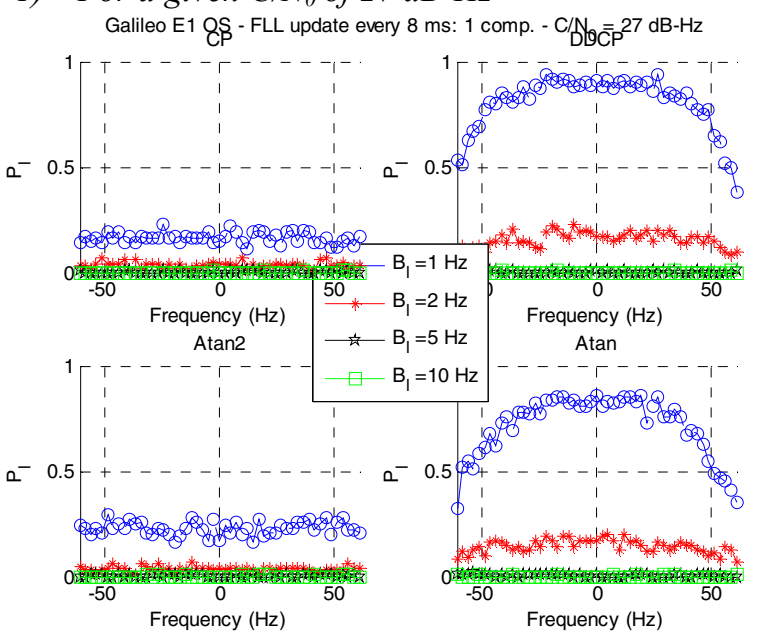

Fig. 15: Results for FLL schemes for Galileo E1 OS at $27 \mathrm{dBHz}$ for the first simulated technique (only one component)

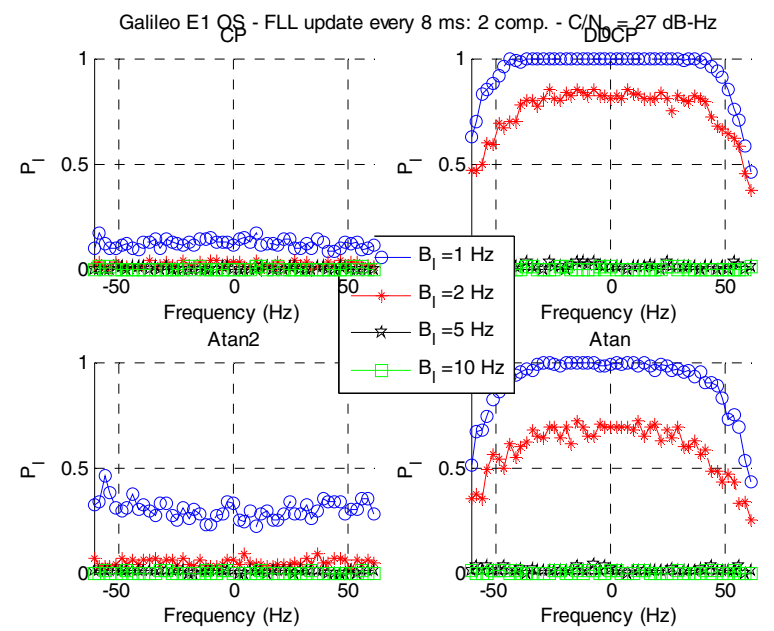

Fig. 16: Results for FLL schemes for Galileo E1 OS at $27 \mathrm{dBHz}$ for the second simulated technique (both component on $8 \mathrm{~ms}$ ) 
Obviously, the probability to get locked is better when both components are used as illustrated in Fig. 15, Fig. 16 and Fig. 17. As previously said for GPS L1 C/A, the best loop bandwidth is $1 \mathrm{~Hz}$ whichever the discriminator. However, for the Galileo E1 OS signal, the best discriminators are the DDCP and Atan. Indeed, the data modulation sensitive discriminators ( $\mathrm{CP}$ and Atan2) suffer from the frequent bit sign transitions on both components (potentially every spreading code).

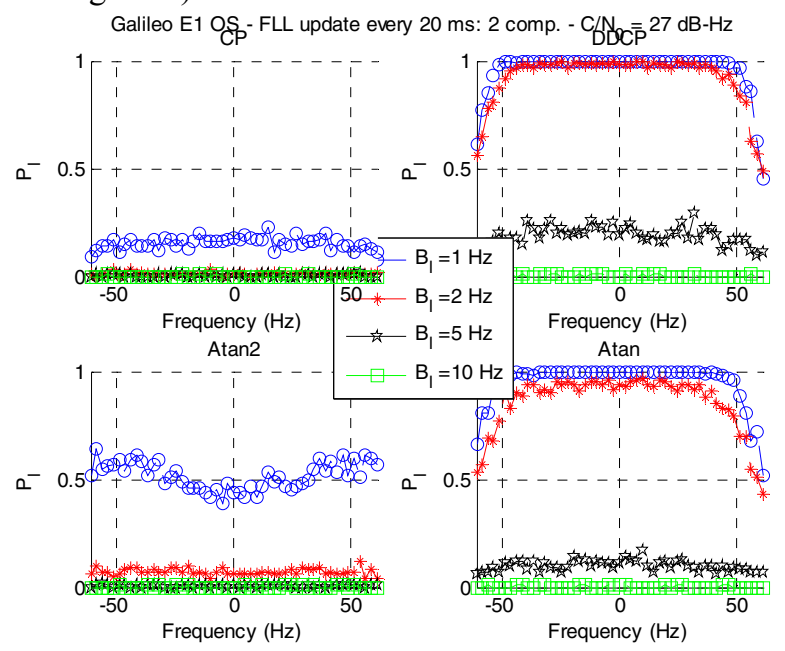

Fig. 17: Results for FLL schemes for Galileo E1 OS at $27 \mathrm{dBHz}$ for the third simulated technique (both components on $20 \mathrm{~ms}$ )

\section{2) For different $C / N_{0}$}

When the probability to get locked is plotted in function of the $\mathrm{C} / \mathrm{N}_{0}$, it can be observed that the Atan 2 discriminator curves (green square in Fig. 19 and Fig. 20) fall for techniques 2 and 3 (on both components), potentially due to bit sign transitions.

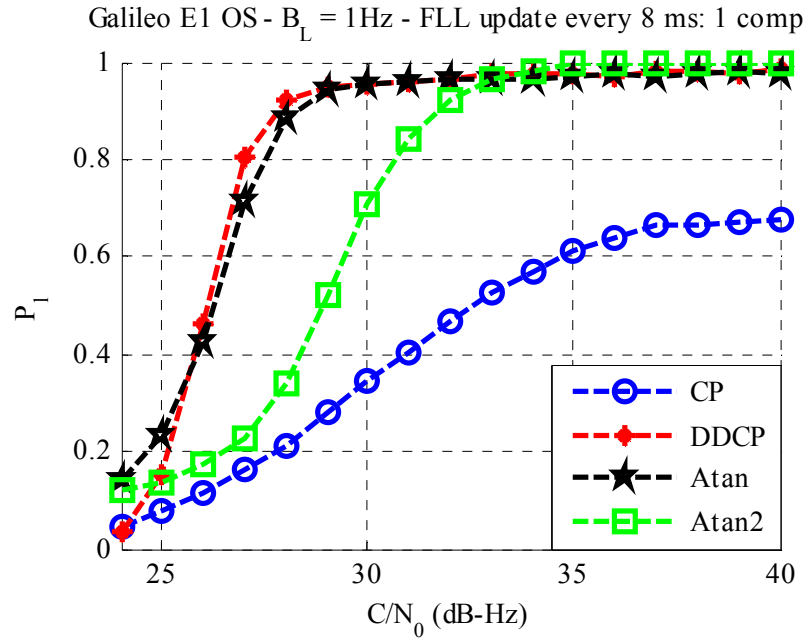

Fig. 18: Results for FLL schemes for Galileo E1 OS for the first simulated technique (one component on $8 \mathrm{~ms}$ )

Between the use of one (Fig. 18) or both components (Fig. 19), there is a difference a gain of 1 or $2 \mathrm{~dB}$ (equivalent $\mathrm{C} / \mathrm{N}_{0}$ ) for the DDCP and Atan discriminators. The gain between technique 2 (on $8 \mathrm{~ms}$ ) or technique 3 (on $20 \mathrm{~ms}$ ) is comparable in the same order ( 1 or $2 \mathrm{~dB})$.

Galileo E1 OS - $\mathrm{B}_{\mathrm{L}}=1 \mathrm{~Hz}-\mathrm{FLL}$ update every $8 \mathrm{~ms}: 2$ comp.

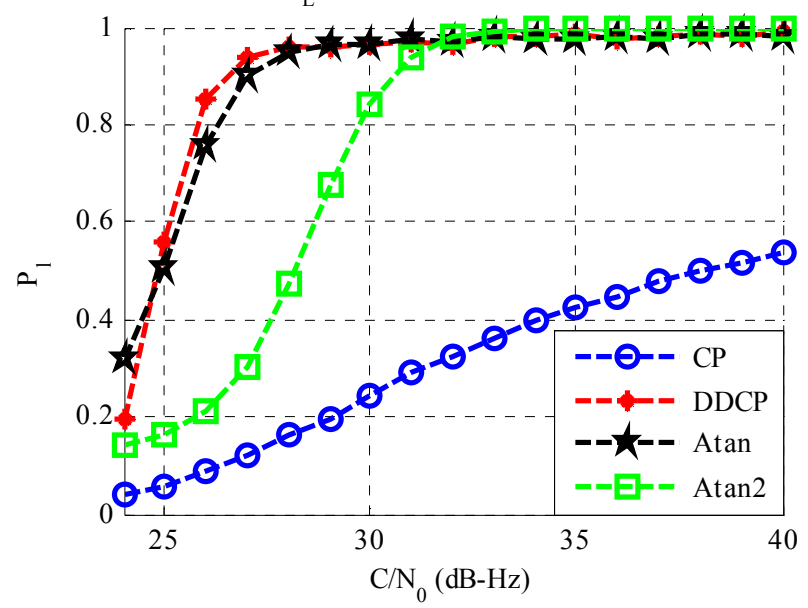

Fig. 19: Results for FLL schemes for Galileo E1 OS for the second simulated technique (on both components on $8 \mathrm{~ms}$ )

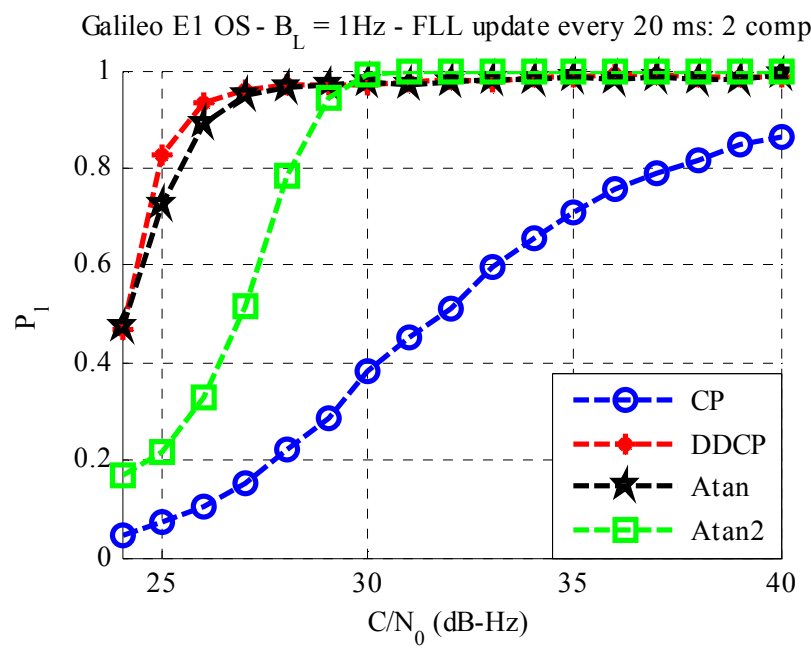

Fig. 20: Results for FLL schemes for Galileo E1 OS for the second simulated technique (on both components on $8 \mathrm{~ms}$ )

At $27 \mathrm{~dB}-\mathrm{Hz}$, the best discriminators are the DDCP and Atan which provides a probability to get locked of 0.8 for the first technique (on one component) and around 0.95 for techniques 2 or 3 (FLL update every $8 \mathrm{~ms}$ or $20 \mathrm{~ms}$ ) which should be preferred.

The data modulation sensitive discriminators (CP and Atan2) performance for the Galileo E1 OS signal is not as good as for the GPS L1 C/A. This is effectively due the presence of frequent bit transitions on the Galileo E1 OS signal. Table I provides the average probabilities to get locked when considering data and secondary code (denoted as Data) or assuming that data and secondary codes are not present (denoted as No data). It can be seen that the presence of bit sign transitions for Galileo E1 OS degrades considerably the probability to get locked. When it equals 1 when it is assumed that no data are present, it falls to 0.2 when data and secondary codes are considered. The same study was done for GPS L1 
$\mathrm{C} / \mathrm{A}$ and it shows that there is no real difference between both cases due to the different signal structure.

TABLE I. AVERAGE PROBABILITY TO GET LOCKED AT 27 DB-Hz IN PRESENCE/ABSENCE OF DATA AND SECONDARY CODE (GALILEO E1 OS)

\begin{tabular}{|c|c|c|c|c|c|}
\hline \multicolumn{2}{|c|}{} & CP & DDCP & Atan & Atan2 \\
\hline \multirow{2}{*}{$\begin{array}{c}\text { Technique } \\
1\end{array}$} & Data & 0.16 & 0.8 & 0.71 & 0.23 \\
\cline { 2 - 6 } & No data & 1 & 0.8 & 0.71 & 1 \\
\hline $\begin{array}{c}\text { Technique } \\
2\end{array}$ & Data & 0.12 & 0.94 & 0.90 & 0.30 \\
\cline { 2 - 6 } & No data & 1 & 0.93 & 0.90 & 1 \\
\hline $\begin{array}{c}\text { Technique } \\
\mathbf{3}\end{array}$ & Data & 0.15 & 0.96 & 0.95 & 0.52 \\
\cline { 2 - 5 } & No data & 1 & 0.96 & 0.95 & 1 \\
\hline
\end{tabular}

\section{CONCLUSIONS}

This paper has investigated the acquisition-to-tracking transition problem through a study on different FLL schemes and for both civil L1 GPS and Galileo signals. This works allows investigating the optimal FLL scheme to optimize the probability that the FLL converges after acquisition for a received $\mathrm{C} / \mathrm{N}_{0}$ equal or above $27 \mathrm{~dB}-\mathrm{Hz}$.

The theoretical study on four popular discriminators reminds their main properties:

- The Cross Product discriminator is not noise dependent which is a considerable advantage for low $\mathrm{C} / \mathrm{N}_{0}$

- $\quad$ The Decision Directed Cross Product and Differential Arctangent discriminators are data modulation insensitive

- The Four-Quadrant Arctangent discriminator is the discriminator which has the biggest linear region

Monte-Carlo simulations permit to assess the FLL scheme performance in terms of probability of successful convergence after 20 seconds. The different FLL scheme parameters that were tested are:

- The discriminator (Cross Product, Decision-Directed Cross Product, Differential Arctangent, FourQuadrant Arctangent)

- $\quad$ The loop bandwidth $(1,2,5,10 \mathrm{~Hz})$

- The correlator outputs techniques:

- GPS L1 C/A: on $2 \mathrm{~ms}$

- Galileo E1 OS: on one or both components on $8 \mathrm{~ms}$

○ GPS L1 C/A: on $20 \mathrm{~ms}$ by averaging 19 discriminator outputs computed every $2 \mathrm{~ms}$

○ Galileo E1 OS: on $20 \mathrm{~ms}$ by averaging 8 discriminator outputs (4 per component) computed every $8 \mathrm{~ms}$

More than 30 FLL schemes for each signal are simulated in order to provide a large comparison performance of FLL schemes to choose the best ones and the probability to converge.
It has been shown that:

- The best loop bandwidth is $1 \mathrm{~Hz}$ (or below). In particular, for the GPS L1 C/A signal, a higher loop bandwidth can be used for the same probability to successful convergence (1) and to converge more quickly.

- For the GPS L1 C/A signal, the best FLL scheme is with a $\mathrm{CP}$ or Atan2 discriminator due to the rarity of the data bit sign transitions (wrt the spreading code duration)

- For the Galileo E1 OS signal, it would be wise to use both components on 4 or $20 \mathrm{~ms}$ but with the DDCP or Atan discriminators.

- For Galileo E1 OS, it seems to be very difficult to succeed the acquisition-to-tracking transition with data modulation sensitive discriminators because the probability to get locked really suffers from the almost constant bit transitions.

The work can clearly be extended by refining this general study. Criteria or constraints on the time to convergence or computational execution cost can be added. Finally, it would be interesting to study the performance of the FLL schemes on real signals to check the theoretical and simulated results presented in this paper.

\section{REFERENCES}

[1] Navstar, "GPS Space Segment/Navigation User Interfaces (IS-GPS-200G)." Sep-2012.

[3] F. Bastide, "Analysis of the Feasibility and Interests of Galileo E5a/E5b and GPS L5 for Use with Civil Aviation," Ph.D. thesis, Institut National Polytechnique de Toulouse, 2004

[4] H. Al Bitar, "Advanced GPS Signal Processing Techniques for LBS Services," Ph.D. thesis, Institut National Polytechnique de Toulouse, 2007.

[5] RTCA, Inc, "Assessment of Radio Frequency Interference Relevant to the GNSS L1 Frequency Band RTCA/DO-235B.” 13-Mar-2008.

[6] J. T. Curran, "A Comparison of Differential-Power and Differential-Phase FLLs for GNSS Receivers," in Proceedings of the 25th International Technical Meeting of The Satellite Division of the Institute of Navigation (ION GNSS 2012), Nashville, TN, USA, 2012, pp. 1477 1487.

[7] J. T. Curran, "Weak Signal Digital GNSS Tracking Algorithms," Ph.D. thesis, National University of Ireland, Cork, 2010.

[8] J.-C. Juang and Y.-H. Chen, "Phase/Frequency Tracking in a GNSS Software Receiver," IEEE J. Sel. Top. Signal Process., vol. 3, no. 4, pp. $651-660$.

[9] J. T. Curran, G. Lachapelle, and C. C. Murphy, "Improving the Design of Frequency Lock Loops for GNSS Receivers," IEEE Trans. Aerosp. Electron. Syst., vol. 48, no. 1, pp. 850-868, Jan. 2012. 
[10] J. J. Parkinson and J. J. Spilker, Global Positioning System: Theory and Applications, Progress in Astronautics and Aeronautics., vol. I, 2 vols. 1996.

[11] E. D. Kaplan and C. Hegarty, Understanding GPS: Principles and Applications, 2nd edition. Artech House, 2005.

[12] P. Ward, "Performance Comparisons Between FLL, PLL and a Novel FLL-Assisted-PLL Carrier Tracking Loop Under RF Interference Conditions," in Proceedings of the 11th International Technical Meeting of the Satellite Division of The Institute of Navigation (ION GPS 1998), Nashville, TN, USA, 1998, pp. 783 - 795.

\section{APPENDICES}

\section{A. Appendix A: Cross Product discriminator}

For simplification, the correlator outputs given in (6) are expressed as the sum of the useful signal and noise (15).

$$
\left\{\begin{array}{l}
I(k)=A_{I}(k)+n_{I}(t) \\
Q(k)=A_{Q}(k)+n_{Q}(t)
\end{array}\right.
$$

1) Cross-product discriminator output noise

The resulting noise $n_{C P}$ of the cross product discriminator output is given by (16) and it corresponds to the sum of the product of the useful parts with a correlator output noise term and the product of the correlator output noise terms.

$$
n_{C P}(k)
$$

$$
\begin{array}{rr}
=\quad & \frac{1}{T_{I}}\left(A_{Q}(k) n_{I}(k-1)+A_{I}(k-1) n_{Q}(k)\right) \\
& -\frac{1}{T_{I}}\left(A_{I}(k) n_{Q}(k-1)-A_{Q}(k-1) n_{I}(k)\right) \\
& +\frac{1}{T_{I}}\left(n_{Q}(k) n_{I}(k-1)-n_{I}(k) n_{Q}(k-1)\right) \\
\text { 2) } & C P \text { discriminator response for GPS L1 C/A }
\end{array}
$$

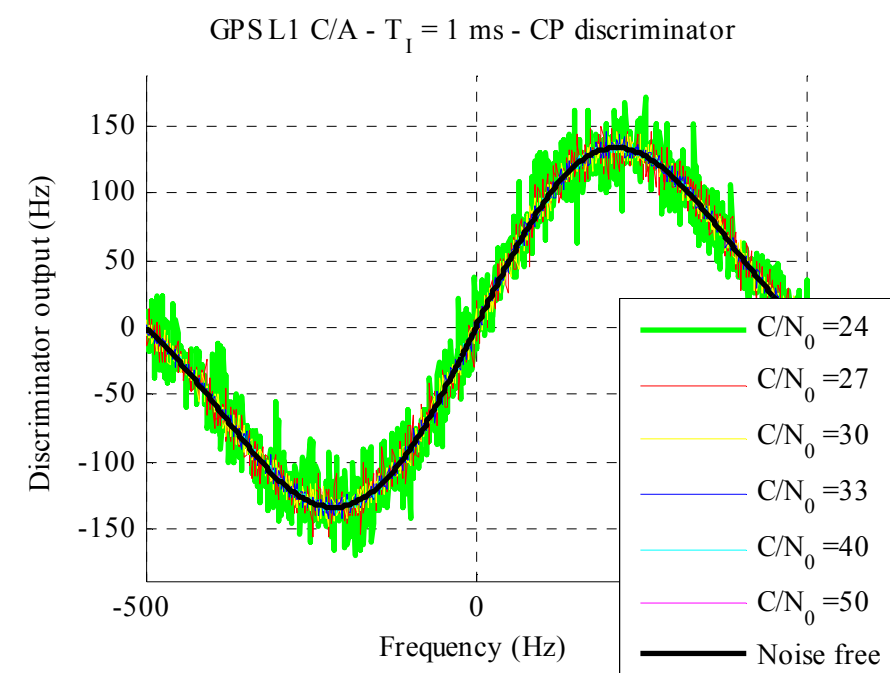

Fig. 21: CP discriminator response to frequency error for a noise-free signal and different $\mathrm{C} / \mathrm{N}_{0}$ cases
B. Appendix B: Decision Directed Cross Product discriminator

1) Mathematical model of DDCP discriminator

The dot product is expressed as (17) assuming no noise. Dot $=I(k) I(k-1)+Q(k) Q(k-1)$

$$
\begin{gathered}
=\frac{A^{2}}{4} d(k-1) d(k) \cos (\phi(k)-\phi(k-1)) \\
\quad \times \operatorname{sinc}\left(\pi \varepsilon_{f}(k) T_{I}\right) \operatorname{sinc}\left(\pi \varepsilon_{f}(k-1) T_{I}\right)
\end{gathered}
$$

Then, the Decision Directed Cross Product is a function of the sign of the dot product which can be seen as (18) by expressing the sign function as $\operatorname{sign}(x)=\frac{x}{|x|}$. The expressions are given without the noise term.

Let us note $\Delta_{\phi}(k)=\varepsilon_{\phi}(k)-\varepsilon_{\phi}(k-1)$

$$
\begin{array}{ccc} 
& \operatorname{sign}(I(k) I(k-1)+Q(k) Q(k-1)) \\
= & \operatorname{sign}\left(d(k-1) d(k) \cos \left(\Delta_{\phi}(k)\right)\right) \\
= & \frac{d(k-1) d(k) \cos \left(\Delta_{\phi}(k)\right)}{\left|\cos \left(\Delta_{\phi}(k)\right)\right|} \\
= & \quad A_{C P} d(k-1) d(k) d(k-1) d(k) \\
\times & \frac{\sin \left(\Delta_{\phi}(k)\right)}{T_{I}} \frac{\cos \left(\Delta_{\phi}(k)\right)}{\left|\cos \left(\Delta_{\phi}(k)\right)\right|} \\
= & A_{C P} \times \frac{\sin \left(2 \Delta_{\phi}(k)\right)}{T_{I} \times\left|\cos \left(\Delta_{\phi}(k)\right)\right|}
\end{array}
$$

2) DDCP discriminator response for GPS $L 1 C / A$ GPS L1 C/A - $\mathrm{T}_{\mathrm{I}}=1 \mathrm{~ms}$ - DDCP discriminator

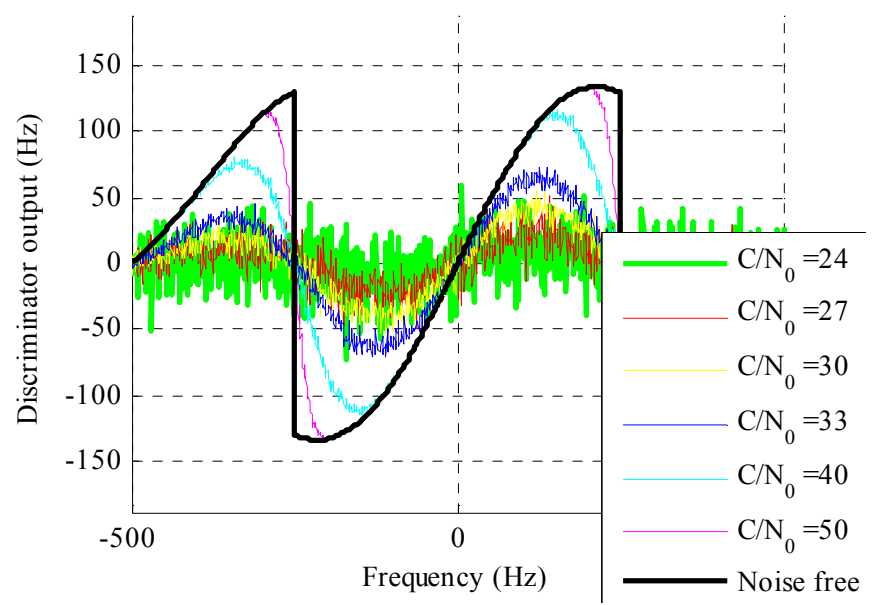

Fig. 22: DDCP discriminator response to frequency error for a noise-free signal and different $\mathrm{C} / \mathrm{N}_{0}$ cases (GPS L1 C/A) 
C. Appendix C: Differential Arctangent Discriminator

The function $U$ is defined as:

$$
U(x)=\left\{\begin{array}{ccc}
x-\pi & \text { for } & x \geq \frac{\pi}{2} \\
x & \text { for } & -\frac{\pi}{2} \leq x \leq \frac{\pi}{2} \\
x+\pi & \text { for } & x \leq-\frac{\pi}{2}
\end{array}\right.
$$

GPS L1 C/A - T $=1 \mathrm{~ms}$ - Atan discriminator

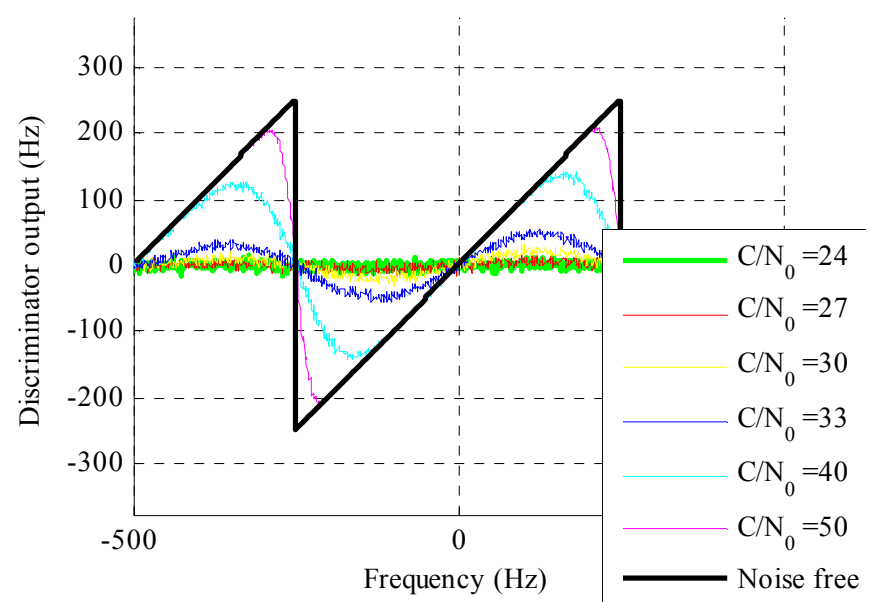

Fig. 23: Atan discriminator response to frequency error for a noise-free signal and different $\mathrm{C} / \mathrm{N}_{0}$ cases (GPS L1 C/A)

D. Appendix D: Four-Quadrant Arctangent (Atan2) Discriminator

1) Mathematical model of Atan2 discriminator

The definition of the Atan2 function is reminded here.

$$
\begin{gathered}
\operatorname{atan} 2(y, x)=2 \times \tan ^{-1}\left(\frac{y}{x+\sqrt{x^{2}+y^{2}}}\right) \\
=\left\{\begin{array}{ccc}
\tan ^{-1}\left(\frac{y}{x}\right) & \text { for } & x>0 \\
\tan ^{-1}\left(\frac{y}{x}\right)+\pi \times \operatorname{sign}(y) & \text { for } & x<0 \\
\frac{\pi}{2} \times \operatorname{sign}(y) & \text { for } & y \neq 0, x=0 \\
\text { undefined } & \text { for } y=0, x=0
\end{array}\right.
\end{gathered}
$$

The term Cross and Dot are previously:

$$
\begin{gathered}
\text { Cross }=A_{C P} d(k-1) d(k) \frac{\sin \left(\varepsilon_{\phi}(k)-\varepsilon_{\phi}(k-1)\right)}{T_{I}} \\
\text { Dot }=A_{C P} d(k-1) d(k) \frac{\cos \left(\varepsilon_{\phi}(k)-\varepsilon_{\phi}(k-1)\right)}{T_{I}}
\end{gathered}
$$

Then without noise $\operatorname{Cross}^{2}+\operatorname{Dot}^{2}=A_{C P}^{2}$. Under the same assumption, it can be shown that the denominator is positive for $\left|\varepsilon_{\phi}(k)-\varepsilon_{\phi}(k-1)\right|<\frac{\pi}{2}$

$$
\begin{gathered}
D o t+\sqrt{\operatorname{Cross}^{2}+\operatorname{Dot}^{2}} \\
=A_{C P}+A_{C P} d(k-1) d(k) \cos \left(\varepsilon_{\phi}(k)-\varepsilon_{\phi}(k-1)\right) \\
=A_{C P}\left(1+d(k-1) d(k) \cos \left(\varepsilon_{\phi}(k)-\varepsilon_{\phi}(k-1)\right)\right)
\end{gathered}
$$

Moreover, the ratio Cross / Dot gives the tangent function of the phase error.

$$
\frac{\text { Cross }}{\operatorname{Dot}}=\frac{\sin \left(\varepsilon_{\phi}(k)-\varepsilon_{\phi}(k-1)\right)}{\cos \left(\varepsilon_{\phi}(k)-\varepsilon_{\phi}(k-1)\right)}=\tan \left(\Delta_{\phi}(k)\right)
$$

2) Atan2 discriminator response for GPS L1 C/A GPS L1 C/A - $\mathrm{T}_{\mathrm{I}}=1 \mathrm{~ms}$ - Atan2 discriminator

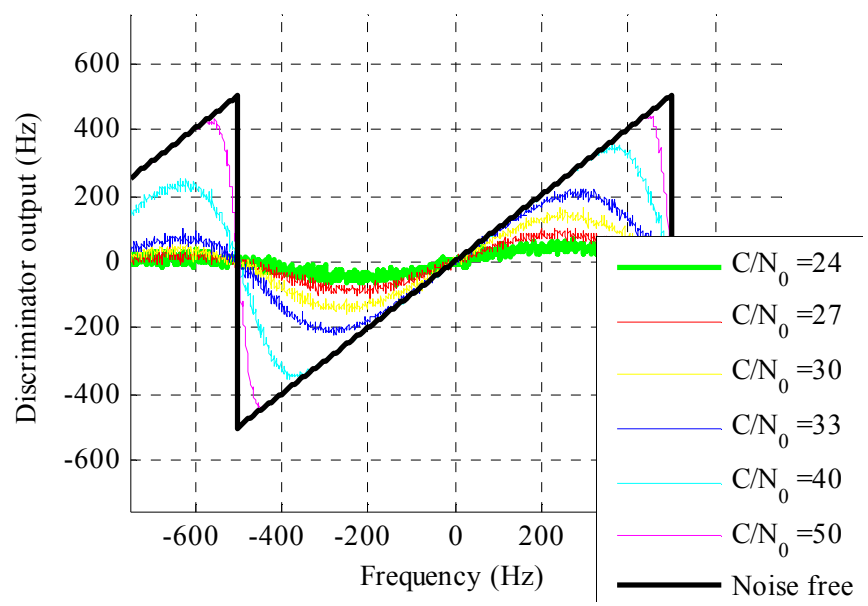

Fig. 24: Atan2 discriminator response to frequency error for a noise-free signal and different $\mathrm{C} / \mathrm{N}_{0}$ cases (GPS L1 C/A) 